This item was submitted to Loughborough's Research Repository by the author.

Items in Figshare are protected by copyright, with all rights reserved, unless otherwise indicated.

\title{
Prediction of the spatial characteristics of higher mode sound transmission through a periodic slit screen
}

PLEASE CITE THE PUBLISHED VERSION

http://dx.doi.org/10.1016/j.apacoust.2014.03.009

PUBLISHER

(C) Elsevier

VERSION

AM (Accepted Manuscript)

LICENCE

CC BY-NC-ND 4.0

\section{REPOSITORY RECORD}

Horner, Jane L., and Stephen J. Walsh. 2014. "Prediction of the Spatial Characteristics of Higher Mode Sound Transmission Through a Periodic Slit Screen”. figshare. https://hdl.handle.net/2134/14486. 
This item was submitted to Loughborough's Institutional Repository (https://dspace.lboro.ac.uk/) by the author and is made available under the following Creative Commons Licence conditions.

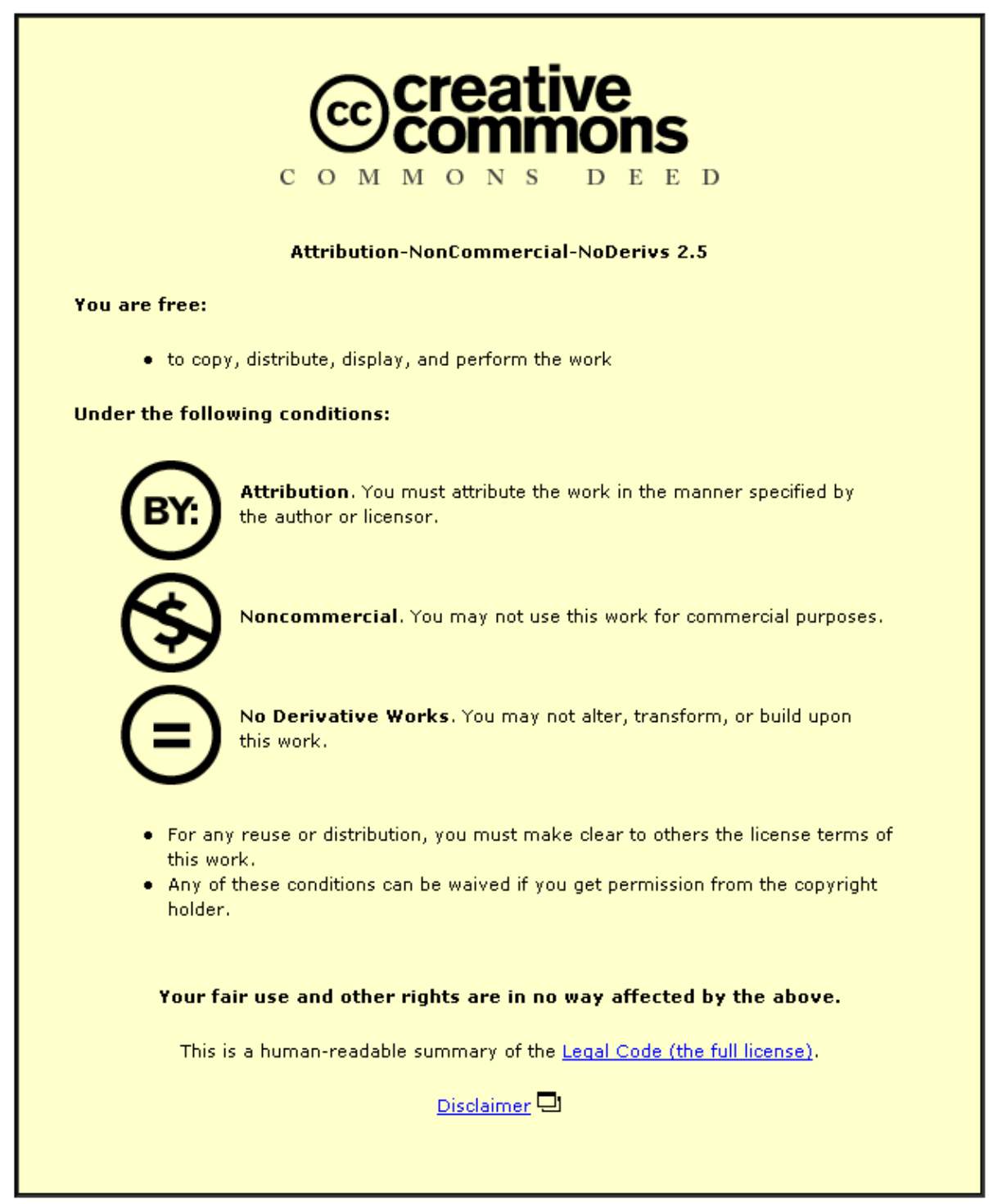

For the full text of this licence, please go to: http://creativecommons.org/licenses/by-nc-nd/2.5/ 


\title{
Prediction of the spatial characteristics of higher mode
}

\section{sound transmission through a periodic slit screen}

\author{
J. L. Horner
}

Department of Aeronautical and Automotive Engineering,

Loughborough University, Loughborough, LE11 3TU, United Kingdom.

S. J. Walsh*

Department of Aeronautical and Automotive Engineering,

Loughborough University, Loughborough, LE11 3TU, United Kingdom

* Corresponding author. Tel.: +44(1509) 227208

E-mail address: S.J.Walsh@Lboro.ac.uk (S. J. Walsh) 


\begin{abstract}
Periodic slit screens are often installed in rectangular apertures either to mask the opening or to reduce transmitted noise. This investigation considers the higher-order scattered and transmitted sound from a point source through a periodic screen mounted in a rigid baffle. In particular, this paper considers the spatial characteristics, or directivity, of the scattered field for a particular higher order mode. Uncoupled higher-order mode analysis is used to estimate the scattered and transmitted sound fields for the selected mode of the aperture without the presence of the screen. Transmission coefficients for the screens are then calculated using the well established equivalent mass layer effect and applied to the calculated higher-order mode scattered and transmitted sound pressures. Using an anechoic chamber, measurements were made over a small arc of the scattered sound field through a range of screens of different aspect ratios but the same porosity. The screens consisted of equally spaced open slits and solid laths and the number of slits was reduced to change the aspect ratio. In each case the point source was positioned on the impinging side of the aperture so as to drive one particular scattered higher-order aperture mode at or near cut-on. Comparison of the measured and predicted sound pressures indicate that good estimates of the sound field can be obtained through the approach of applying simple corrections for the presence of the screens to the estimates for the open aperture established using uncoupled calculations for the higher-order modes. It is also shown that the results for a specific mode excited at one particular frequency are applicable at other excitation frequencies provided that the correct nondimensionalisation is applied.
\end{abstract}




\section{Introduction}

One of the most common problems in engineering applications is the transfer of acoustic energy from one domain to another via an opening in a hard surface. At low frequencies, when the wavelength of the incident wave is much greater than the aperture dimension, well-established approximate methods may be used to estimate the transmission loss. When the wavelength of the incident wave field approaches the dimensions of the aperture, higher-order modes will propagate in the aperture and estimates of transmission loss must include contributions from these modes. As cross coupling contributions between higher-order modes must be calculated, this is a numerically intensive technique that does not yield a simple solution. In many situations a device may also be inserted in the aperture to either mask the opening or to reduce transmitted noise. The presence of the device increases the complexity of the fully coupled analysis for higher order mode transmission. When considering rectangular apertures, one of the most common types of device is the periodic slit screen consisting of equally spaced solid, or lath, and open slit. This type of screen is usually installed to mask an open aperture and well-established techniques exist to determine the transmission coefficient of such screens.

Saunter and Soroka [1] developed an approximate expression for sound transmission for rectangular apertures between reverberant enclosures based on the piston impedance functions for circular pistons. Due to the approximations used for the rectangular analysis, the expressions were limited to apertures of aspect ratio of eight or less. Other approaches [2-4] investigated apertures with greater aspect ratios that may be considered as slits and developed expressions for transmission loss that demonstrated the relationship between aperture dimensions and frequency 
characteristics. In particular Mechel [4] developed analytical expressions for the oblique incidence transmission loss of slits.

Once the wavelength of the incident wave approaches the depth of the aperture, higher order duct modes will propagate in the aperture. Thus, full solutions to the transmission problem must include coupling between the higher-order modes [5] that is a computationally intensive process if a full numerical solution is used. Previous work [6-8] has evaluated the fully coupled problem for plane wave impingement over a wide frequency range. Sgard, Nelisse and Atalia [9] presented a comprehensive review of the various approaches to modelling of sound transmission through finite thickness apertures. This review concluded that many of the existing models that consider the effect of higher-order modes in the aperture are too time consuming to implement. Analysis was then presented for modelling diffuse field sound transmission that includes higher order contributions. In reference [9] it was noted that only including modes up the maximum frequency of interest was sufficient to capture the sound field. It was also noted that there was strong cross modal coupling between the $(0,0)$ plane wave mode and higher order even-even modes and that for aspect ratios greater than one, the transmission models based only on plane waves may not be sufficient even at low frequencies, due to the low values for the cut-on frequency of the first higher order mode. Sgard and co-researchers [10] continued the analysis by performing experimental validation tests. Results were presented for both rectangular apertures and slits and good comparison was found between measurements on apertures and estimated results. Caution was expressed over the measured data for slits due to the problem of flanking transmission in the experimental set-up. 
Sound intensity techniques have been used to determine the transmission loss of slit shaped apertures in rigid walls separating two reverberant enclosures [11]. Good agreement was found between measured transmission loss and the value determined using the Gomperts-Kihlman [3] approach in the frequency region were the product of the wave number and the width of the slit was less than two. It was shown that for a given slit depth, the difference between measured and calculated values of transmission loss increased as the width of the slot decreased due to the effects of viscosity and friction. Ref. [11] also commented on the difference between measured and calculated values for wider slits due to the propagation of the higher order modes in the test aperture, which were not accounted for in the analysis.

The fully coupled solution developed by Park and Eom [8], used the Fourier Transform and mode matching techniques to obtain a rigorous solution to the fully coupled problem. Horner and Peat [12] used this approach to determine a simple approximate solution for the scattered sound field resulting from plane wave impingement on a rectangular aperture in a rigid baffle separating two semi-infinite half spaces when the frequency of excitation is such that higher-order modes propagate in the aperture. Attention concentrated on the cross-coupling effects between higher-order modes and the development of approximate expressions to describe the propagating higher-order modes. All analysis was undertaken in nondimensional wave number space to allow results to be applicable to any combination of aperture size and input frequency. Different in-aperture modes were analysed by keeping the excitation frequency fixed but moving the location of the excitation so as to excite the particular mode of interest. Results demonstrated that when the propagating scattered field of the higher order modes are presented in nondimensional format, all the modes follow similar trends with the largest response 
occurring approximately at the cut-on frequency of the mode. Thus, rather than considering the sound scattered from the aperture as a function of frequency, it was possible to keep frequency constant and consider the spatial responses or directivity of different modes. The investigation considered the contribution from the crosscoupling terms to the propagating higher-order modes and demonstrated that good approximations for the cut-on modal contributions can be obtained from uncoupled calculations provided at least twenty five in-aperture modes were cut-on in the aperture at the frequency of interest. Using uncoupled calculations, which ignore the cross coupling contributions between modes, resulted in a significant saving in the computational time required to obtain an equivalent solution using a fully-coupled wave analysis. This analysis was extended to point source excitation [13] and estimated values were compared with measured data. Initially the source was located at the specific position required to drive a selected scattered higher-order mode at its cut-on frequency. The source was then located at positions that did not drive any mode exactly at cut-on. Good comparisons between the uncoupled solutions and the measured scattered field were obtained for modes not driven at the corresponding cuton frequency, as well as modes driven at cut-on, which established that the method can be used for any relative location of the source from the aperture. Alternatively, it could be considered that this meant that the approximations from uncoupled calculations could be used over a wider frequency range for a given fixed location of the source, subject to a limit on the minimum number of cut-on in-aperture modes present in the aperture. As for both the plane wave and the point source approximations, it was assumed that a significant number of modes were cut-on in the aperture at the frequency of interest thus negating consideration of the contributions from the evanescent or cut-off modes. 
Well established techniques [14] exist to determine the transmission coefficient for flat plates, or screens, with periodic slits. The mass layer effect of the slits may be calculated using the dimensions of the slits and the screen. End corrections must be applied to the depth of the slit and these are a function of the distance between the plate with slits and any backing plate. If no backing plate is present, the end-correction for the largest distance should be used. This approach was applied in the analysis of a barrier with regular rectangular slit-like openings [15]. Although a barrier rather than a screen with slits was considered, the analysis of the transmission through the regular openings used Fresnel-Kirchhoff diffraction theory and then represented the barrier as a partially transmitting continuous screen with an effective surface mass density. Results showed that the approach was not applicable at lower frequencies when the wavelength was greater then the width of the solid as the Fresnel-Kirchhoff diffraction theory could not be applied. A similar approach [16] was used for the transmission through acoustic louvres, at low frequency when the performance of the louvre is dominated by the openings in the device rather than the presence of the blades. The louvre was also modelled as a thin screen with multiple slits, using the Fresnel-Kirchhoff diffraction equation and was assumed to be a partially transmitting solid screen with an effective surface mass density related to the air in the slits. Results showed that the prediction underestimated the measured transmission loss and that the model was inappropriate for oblique gaps to the surface which were long compared to the wavelength

In this investigation, measurements have been made, using an anechoic chamber, of the higher-order scattered and transmitted acoustic fields through a rectangular aperture containing a periodic slit screen. Nearly all measurements have been made at a single frequency, $1 \mathrm{kHz}$. However, it will be demonstrated, with 
additional measurements made at 2, 3, 6 and $8 \mathrm{kHz}$, that when the data are represented in appropriate non-dimensional form then the cut-on scattered modes follow the same directivity characteristic, irrespective of the driving frequency. Hence, in the investigation of the slit screen investigation the frequency is kept constant and the different modes are investigated by changing the position of the source such that the desired mode is cut-on. Due to the non-orthogonal nature of the higher-order modes in rectangular cross-section apertures, small contributions from all cut-on modes are present at all frequencies, but have been shown to be negligible [12] if a mode is excited at or close to cut-on. For the range of aperture sizes tested the non-dimensional Helmholtz number $k a$, were $k$ is wave number and $a$ is a dimension of the aperture, varied from 3.20 to 9.6, which was equivalent to a frequency range of $330 \mathrm{~Hz}$ to $1 \mathrm{kHz}$ for a fixed size aperture of the largest dimension tested. The aperture was in a rigid baffle and the screen was the same thickness as the baffle and, thus, installed flush to both faces. In each case a point source was used to drive a particular scattered higher-order mode either at cut-on or close to cut-on by selection of the correct source coordinates. The scattered and transmitted sound pressure level from an equivalent size fully open, or empty, aperture was estimated using the approximate uncoupled mode analysis developed in Ref. [13]. Then the transmission coefficient for the periodic slit screen was calculated using the equivalent mass layer effect and used to correct the calculated transmitted sound pressure level for the empty aperture. Thus, the novelty of this work is that established techniques for calculating the transmission coefficient of periodic slit screens, have been applied to the sound pressure level estimated using approximate uncoupled higher-order mode analysis in order to estimate the higher-order sound pressure level transmitted through a periodic slit screen in an aperture. This extension 
of previous work on approximations for higher-order modes in apertures without screens is of interest in order to determine if the uncoupled approach is robust enough to be applied to apertures with screens present. In particular, to establish the quality of the estimates of the spatial response of the scattered field. If the uncoupled analysis cannot be successfully applied to what is, in acoustic analysis terms, a simple screen it is unlikely that the approach can be applied to more complicated devices. Most applications of the mass layer approach are constrained to plane wave propagation only and those that have combined the mass layer approach with other diffraction theories have found the application to be limited. This approach allows the finite thickness of the aperture to be accounted for unlike the Kirchhoff diffraction theory used in previous investigations. The approximations developed for just the aperture assume that coupling effects are minimal and this investigation will determine if that assumption is still valid when a simple periodic screen is placed in the aperture. The device would be expected to increase the level of coupling in the aperture and also the contribution from the evanescent modes. For a periodic slit screen, the velocity decreases inversely with the distance from the centre of the slit [14], thus, increasing the influence of neighbouring slits. Emphasis is placed on the spatial characteristics of the results for the scattered field as this is the most numerically intensive part any full calculation and any estimate must successfully capture the spatial trends in order to estimate the directivity of the higher order mode.

As noted above, it will also be demonstrated using estimates that the spatial characteristics of the scattered field from an open aperture are independent of the driving frequency and are a function of the mode number and aperture dimensions. Due to the practical limitations of the size of the experimental facility only the $(1,0)$, $(2,0),(0,1)$ and $(2,1)$ modes were investigated experimentally, where for a mode, $(m$, 
$n), m$ denotes the number of nodal lines in the major aperture dimension and $n$ the number of nodal lines in the minor aperture dimension. However, this set of modes did allow both axial and tangential in-aperture modes to be investigated. The $(2,1)$ mode was of particular interest as the resulting spatial patterns for the mode was not symmetric to the aperture axis and the ability to successful capture the secondary "peaks" of the spatial response was necessary for the correct estimate of directivity. Thus of particular interest is the quality of the estimate of the spatial characteristics of the scattered field, which becomes more complex with increasing mode number. The set of modes investigated also allowed one even-even mode to be investigated, noting the comments in Ref. [9] on the strong coupling of even-even modes to the $(0,0)$ plane wave mode.

The slit screens were oriented such that the laths were parallel to the nodal lines of the $(1,0)$ and the $(2,0)$ modes and for certain aperture dimensions the dominant "line of sight" transmission path from the source through the centre of the aperture to the microphone was blocked by a lath. Thus, the laths were perpendicular to the $(0,1)$ nodal lines and could be considered to cut across the nodes. Six different aperture aspect ratios in the range 1.45 to 4.35 were tested, which corresponded to a Helmholtz number range of 3.2 to 9.6 for the largest aperture. This is within the range of interest for most noise control applications, which is usually taken to be Helmholtz Numbers between 1 and 10. In each case the slit screen had the same porosity but the number of laths and slits reduced as the aspect ratio was increased. This also allowed consideration of the effect of reducing the number of cut-on modes in the equivalent open aperture and the positioning of a lath either on or not on the direct line of sight path from the source to the receiver. The number of cut-on in-aperture modes at the test frequency ranged from nineteen to fifty, with the nineteen modes being less than 
the advised twenty five mode limit [12] on the uncoupled higher-order mode analysis technique. Thus, as the number of cut-on modes reduced, the relative contribution from the evanescent modes increased, the effect of which are not accounted for in the approximate calculations.

As each mode is approximated as being uncoupled from all other modes, frequency domain calculation could be carried out by calculating each modal contribution over the frequency range of interest and then summing the modes to obtain the whole field. Errors will be introduced if the frequency range contains cutoff modes as the evanescent contribution from these modes are not accounted for in the uncoupled estimate. If the spatial characteristics of the individual mode are inaccurate, then the errors would magnify in the overall field calculation. It should be noted that using the simple mass layer approach to estimate the effect of the screen would limit the upper frequency range of the application since it is not valid once diffraction effects occur in the screen itself.

\section{Acoustic fields and analysis}

The following analysis for an open aperture is based on that given by Park and Eom [8] for an incident plane wave on an aperture in a rigid wall. This analysis was extended by Horner and Peat [13] who considered a point source of excitation and also introduced non-dimensional parameters. The non-dimensional depth ratio parameter used is similar to that employed by Gomperts and Kihlman [3]. Given below are the subsequent approximations for estimating the scattered field. Full details of the analysis and the underlying approximations are given in Refs. $[8,12,13]$. 
Consider a rectangular aperture of height $2 a$, width $2 b$ and depth $d$ in a rigid wall (Fig. 1). The velocity potential $\Phi^{p}$ for a spherical wave from the point source $P$ of wave number $k$ may be written as [13]

$$
\Phi^{p}(x, y, z)=\frac{a}{\left|\underline{R}_{p}\right|} e^{i k\left|\underline{R}_{p}\right|}
$$

where $\underline{R}_{p}$ is the vector from point source $P$ to general position $(x, y, z)$. The time base of $e^{-i \omega t}$ is suppressed for brevity.

The following is the approximate expression for the transmitted velocity potential $\Phi^{t}[12]$ where $\zeta$, and $\eta$ are the recoil wave numbers and $\gamma_{m n}$ may be considered the forcing function for the mode and determined using the expressions in Ref.[12]:

$\Phi^{t}(x, y, z)=\frac{i}{8 \pi^{2}} \sum_{m=0}^{\infty} \sum_{n=0}^{\infty} \frac{\gamma_{m n}\left(\xi_{m n} a\right)}{\varepsilon_{m} \varepsilon_{n}\left[\sin \left(\xi_{m n} d\right)+i \cos \left(\xi_{m n} d\right)\right]} \int_{-\infty}^{\infty} \int_{-\infty}^{\infty} \frac{1}{(\kappa a)} G_{m}(\zeta a) G_{n}(\eta b) e^{-i[\zeta x+\eta y+\kappa(z+d)]} d(\zeta a) d(\eta b)$

where the function

$G_{m}(u)=\frac{u\left[(-1)^{m} e^{i u}-e^{-i u}\right]}{u^{2}-(m \pi / 2)^{2}}$

and $\varepsilon_{0}=2, \varepsilon_{1}=\varepsilon_{2}=\ldots=1$. The scattered Helmholtz number $\kappa a$ and the in-aperture axial Helmholtz number $\xi_{m n} d$ are given by $(\kappa a)=\sqrt{(k a)^{2}-(\zeta a)^{2}-(\eta b A)^{2}}$ and $\left(\xi_{m n} d\right)=D\left(\xi_{m n} a\right)=D \sqrt{(k a)^{2}-\left(\frac{m \pi}{2}\right)^{2}-\left(\frac{n \pi A}{2}\right)^{2}}$

where the non-dimensional parameters are the aspect ratio $A=a / b$ and the depth ratio $D=d / a$ and $m$ and $n$ are the mode numbers. To obtain good approximations using the 
above uncoupled mode approach to determine the scattered field a significant number of in-duct modes must be cut-on in the aperture, as discussed in detail in Ref. [12].

If the source is assumed to be relatively far from the aperture then at a position close to the source the acoustic potential is essentially that of the point source only as the contributions from the reflected and scattered fields will be negligible. Let the acoustic pressure at a small distance $\left|\underline{R_{p}}\right|$ from the source be $p^{p}$ and the acoustic pressure at some general point in the transmitted field be $p^{t}$, then

$$
p^{t} \approx p^{p} \Phi^{t} / \Phi^{p}
$$

Thus, if the acoustic pressure is measured at a small distance $\left|\underline{R_{p}}\right|$ from the source then the acoustic pressure at any location in the transmitted field can be evaluated from Eqs. (1), (2) and (3). As the pressure is calculated for each mode of interest, the uncoupled estimates of the acoustic pressure could simply be summed to obtain a frequency domain estimate of the transmitted sound. It must be noted that all of the above analysis assumes that a significant number of modes are cut-on in the aperture and that no consideration is given to the contribution from the cut-off evanescent modes. Therefore, this limits the upper frequency range of any estimate of the overall frequency response of an aperture.

It is possible to select the position of the source such that the source is located to drive a scattered mode $(m, n)$ in the transmitted field at cut-on and hence the sound pressure will be a maximum for that mode. Obviously for the scattered mode $(m, n)$ to be cut on, the equivalent $(m, n)$ in-aperture mode must also be cut on. Before the first higher-order mode scatters, the spatial solution for the plane wave shows little variation with angle. Once the higher-order modes cut-on and scatter the spatial pattern becomes more complicated resulting in a directivity pattern with distinct 
lobes. It may be shown [17] that that the largest response for a scattered mode occurs at cut-on when $k_{x} a=m \pi / 2$ and $k_{y} b=n \pi / 2$, where $k_{x}$ and $k_{y}$ are components of the free wave number $k$. As the components of $k$ are also given by Ref. [8] as $k_{x}=$ $k \sin \theta \cos \phi, k_{y}=k \sin \theta \sin \phi$, and $k_{z}=k \cos \theta$, then this implies that the scattered field will be a maximum when $k \sin \theta \cos \phi=m \pi / 2 a$ or $k \sin \theta \sin \phi=n \pi / 2 b$. Thus, for the transmitted scattered field to be a maximum for a mode $(m, n)$, the source must be located at the following angles relative to the centre of the aperture: $\phi=\tan ^{-1}(a n / b m)$ and for $m=0, \theta=\sin ^{-1}(n \pi / 2 b k \sin \phi)$ and for $m>0, \theta=\sin ^{-1}(m \pi / 2 a k \cos \phi)$.

Thus, the above equations can be used to determine the coordinates for the source to be positioned to excite a particular mode $(m, n)$ at its maximum possible scattered amplitude. Also when one higher-order mode is excited at its scattered cuton wave number the contributions from all other higher-order modes are minimal [12] due to the spatial properties of the modal forcing function $\gamma_{m n}$. Thus to drive a particular higher order mode at the maximum scattered response the source must be located at the following Cartesian source coordinates where $z_{s}$ is the perpendicular distance from the aperture centre: $x_{s}=z_{s} \tan \theta \cos \phi, y_{s}=z_{s} \tan \theta \sin \phi$ and the source will located a radial distance $r_{s}$ from the aperture with $r_{s}$ being given by $r_{s}=\left(x_{s}^{2}+y_{s}^{2}+z_{s}^{2}\right)^{0.5}$

For a fixed source location, the scattered field for an aperture may be calculated as a function of both frequency and space, with increased transmission occurring at the higher-order scattered mode cut-on frequencies. Alternatively, the frequency of excitation may be kept constant and the source moved to a different location in order to cut-on a particular mode, as discussed above. Hence, the spatial pattern of the scattered mode of interest can be determined. In this case, the spatial location of maximum sound pressure level for a mode in the source field will occur in 
the direction of the reflected wave [5] and the spatial location of the maximum scattered sound pressure in the transmitted field will occur at the transmitted angle which is the angle of the direct "line-of-sight " from the source through the centre of the aperture to the transmitted field. Fig. 2 shows the scattered sound pressure level calculated for an open aperture of dimensions $a=761 \mathrm{~mm}, b=525 \mathrm{~mm}$ and $d=54$ mm, using the approximate expressions Eqs. (1), (2) and (3) for the $(2,0)$ mode for the three different frequencies $1 \mathrm{kHz}, 2 \mathrm{kHz}$ and $3 \mathrm{kHz}$ using the same source position and receiver locations. The source is located at $x=1.45 \mathrm{~m}, y=0.0$ and $z=6.3 \mathrm{~m}$ and the receiver is located at a radius of $4.7 \mathrm{~m}$ from the centre of the aperture and at a height of $0.57 \mathrm{~m}$. 'Angle' refers to the angle of the receiver.

Clearly each mode has a maximum at 0 degrees, the "direct line of sight" angle for the $(2,0)$ mode, but very different spatial patterns. However, comparing the sound pressure levels at the same source position and receiver locations for different frequencies is not comparing similar acoustic fields as the wavelengths and hence the wave number of the three responses are different. Fig. 3 shows the same mode and the same frequencies, but now both the source locations and the receiver positions for each mode has been recalculated to take account of the change in frequency i.e. the source is relocated to the position to drive the scattered mode at cut-on for that frequency. Thus the product of wave number and source radius, $k r_{s}$, is different for each of the three frequencies as source co-ordinates $\left(x_{s}, y_{s}, z_{s}\right)$ are different. As the source has been located in a new position at each frequency, the relative receiver position must also change to ensure that the receiver is located at the same relative point in non-dimensional wave number space. So the radial position of the receiver must also be scaled by the same amount as the source location change. In Fig. 3, the sound pressure level is plotted against the product of wave number and the sine of the 
receiver angle $\phi$ to demonstrate that the responses for the same mode at three different frequencies are the same, once the source is located at the correct cut-on position for the mode and frequency. Sound pressure level has been normalized by correcting the sound pressure level of the $2 \mathrm{kHz}$ and $3 \mathrm{kHz}$ data by the difference between the maximum value of sound pressure level at that frequency and the maximum value of the $1 \mathrm{kHz}$ sound pressure level. This in essence corrects the sound pressure level of the source at $1 \mathrm{~m}$ in order that the same peak value occurs at each frequency. The slight discrepancies in the overlaying figures are the result of the data point spacing not being the same between the three frequencies after the data has been corrected. To further illustrate the point, Fig. 4 shows the response of the tangential mode $(2,1)$ at the same three frequencies. This mode has the maximum spatial response shifted from $k \sin \phi=0$ as neither $m$ nor $n$ are zero. It may be observed that the key secondary peak is the same for all three frequencies. Thus it has been demonstrated that results obtained at one frequency are applicable to the same higher order scattered mode at another frequency due to the inherent linear relationships between the modes. The directivity pattern of the scattered mode is dependent on the mode numbers and the aperture dimensions, rather than the frequency of excitation, as is demonstrated in the results presented in Figs. 2 and 3.

To further demonstrate the relationship between the modes, Fig. 5 shows the measured sound pressure level at two different frequencies for the $(1,2)$ mode through an aperture of dimensions $a=80.0 \mathrm{~mm} b=79.5 \mathrm{~mm}$ and $d=16 \mathrm{~mm}$. The experimental approach for the measurement is the same as that detailed in Section 3 with the exception that this aperture was in the middle of a large screen installed in the anechoic chamber, rather than in a screen mounted between the doors of the anechoic chamber and free space. This meant these measurements where made with 
the source and receiver located closer to the screen than the other tests but this was compensated for by measuring at a higher frequency meaning the number of wavelengths between source and screen were of a similar order of magnitude. Measurements were made at both $6 \mathrm{kHz}(k a=8.8)$ and $8 \mathrm{kHz}(k a=11.7)$ using an aperture of significantly different dimensions to the rest of the experimental investigation to demonstrate the inherent nature of the modes. In the measurements in Fig. 5 the source has been located at the relevant position in order to excite the $(2,1)$ mode at cut-on for both frequencies. Hence the source is located at two different positions, one for $6 \mathrm{kHz}$ and one for $8 \mathrm{kHz}$. As with the previous figures, the sound pressure level at $8 \mathrm{kHz}$ has been corrected by the difference between the maximum value at $8 \mathrm{kHz}$ and the maximum value at $6 \mathrm{kHz}$. As with Figs. 3 and 4 this corrects the $8 \mathrm{kHz}$ data as if the source gave the same peak value as the $6 \mathrm{kHz}$ data and data is plotted against $k \sin \phi$. Measurements were taken at 5 degree intervals resulting the measured data having a lower resolution and hence a less detailed directivity pattern than the estimated results shown in Figs. 3 and 4. The measured results further demonstrate that the same non-dimensional response for a mode may be obtained at two different frequencies provided the source is located at the correct position. So in the next section, results will be measured at a single frequency and the source position changed to investigate the different modes.

To determine the effect of a periodic slit screen, the equivalent mass layer $m$ for a screen may be calculated using the following expression [14, Eq. (9.21)], where $\rho$ is density, $\sigma$ is porosity, $l_{o}$ is the depth of the slit and $\Delta L$ is the end correction:

$$
m=(\rho / \sigma)\left(l_{o}+2 \Delta L\right)
$$

Porosity is the ratio of slit width divided by the sum of slit width plus lath width. To determine the end correction $\Delta L$ the table in Ref. [14] may be used. Eq. (4) assumes 
that the screen openings are small compared to the acoustic wavelength. The transmission coefficient $\tau$ for an infinite screen may be calculated from Ref. [14] as:

$$
\tau=\left[1+(\omega m \cos \theta / 2 \rho c)^{2}\right]^{-1}
$$

where $\theta$ is the angle of incidence, $m$ is the equivalent mass layer, $\rho$ is the density and $c$ the wave speed. This may then be used to correct the acoustic pressure obtained from Eq. (3). Both Eqs. (4) and (5) are based on plane wave impingement on the screen. There are established limitations to the use of Eq. (5) as discussed in Ref. [14]. For example, using the simple approach of the equivalent mass layer means that there is no consideration of the mode-lath interaction. However, taking this approach rather than modelling the periodic screen as a series of multiple apertures is significantly more computationally effective although it effectively ignores interference effects.

\section{Experimental set-up}

As with the previous experimental investigation [13], to achieve the desired conditions of a point source exciting an aperture separating two free fields, a loudspeaker within a fully anechoic chamber was used as the source. In this investigation, the loudspeaker was suspended on a pulley system that allowed it to be located anywhere in a plane approximately $4.8 \mathrm{~m}$ in the vertical or $x$-direction by 2.7 $\mathrm{m}$ in the horizontal or $y$-direction. By suspending the source in this manner it was possible to use two different source locations for the $(1,0)$ and $(2,0)$ modes i.e. source located below the centre line of the aperture or above the centre line. The source plane was located $6.3 \mathrm{~m}$ (z-direction) from the impinging face of the aperture. This was the greatest possible distance from the aperture that could be obtained in the anechoic 
chamber and was considered to satisfy the condition that the separation between the source and the aperture is sufficiently great that close to the source the measured sound pressure will be effectively the sound pressure of the source. The locations for the speaker were selected for each mode by establishing the coordinates required to position the speaker to drive the scattered mode of interest at cut-on. These coordinates may be determined using the equations given in the previous section

Using the same approach as in previous tests [13], the large double-doors into the anechoic chamber from the outdoors were kept open and a rigid baffle plate was constructed in the rectangular doorway. This arrangement prevented any sound propagating around the sides of the baffle and, hence, minimised flanking transmission. The baffle plate was constructed of a rigid foam core with plastic covering on both sides that gave a total baffle thickness of $54 \mathrm{~mm}$. A rectangular aperture of size $1.52 \mathrm{~m}$ by $1.05 \mathrm{~m}$ in the $x$ and $y$ directions was left in the baffle plate. This open aperture could then be filled by equally spaced strips of solid material of height $1.52 \mathrm{~m}$, width $70 \mathrm{~mm}$ and thickness $54 \mathrm{~mm}$, made from the same materials as the baffle plate. The strips were aligned in the $y$-direction and were spaced at $70 \mathrm{~mm}$ intervals. Thus, the full size aperture of dimensions $1.52 \mathrm{~m}$ by $1.05 \mathrm{~m}$, could be converted into a periodic slit screen of aspect ratio 1.52 by inserting seven solid sections each of width $70 \mathrm{~mm}$, spaced $70 \mathrm{~mm}$ apart i.e. 8 slits and 7 laths. Care was taken to ensure that each strip was positioned flush with the face of the baffle plate and that there were no voids around the edges of the slits. By inserting additional strips of solid material into the slits at the extreme edges of the screen it was possible to reduce the dimension $2 b$ of the screen and hence to increase the aspect ratio to a maximum of 4.35 for a screen consisting of three slits and two laths. In all cases the dimensions of the individual slits and laths were kept constant resulting in each 
different aspect ratio screen having the same porosity of 0.5 [14]. This could be considered as a very high level of porosity and at the limit of using the equivalent mass layer approach. Reducing the porosity to say 0.10 would have resulted in an order of magnitude increase in the equivalent mass effect. However, the main interest in the investigation was the effect on the estimates of the spatial characteristics of the scattered field and for this reason it was decided to keep an equal slit-lath ratio for all tests. Thus, for the different aspect ratios tested, the aperture dimension $2 a$, parallel to the slit major dimension, was kept constant at $1.52 \mathrm{~m}$ but the dimension $2 b$, perpendicular to the slit major dimension, reduced as the number of slits reduced. Sound level meters were used to measure the sound pressure level at a set distance from the loudspeaker source inside the anechoic chamber and also the transmitted sound field on the outdoor side of the baffle plate. The transmitted field measurements were made over an arc of radius $4.73 \mathrm{~m}$ in a horizontal plane centred on a vertical line through the centre of the orifice, at an above ground height of $1.0 \mathrm{~m}$ $(x=-0.57 \mathrm{~m})$. Previous experimental investigations with a point source [13] for a similar arrangement for the open aperture had taken measurements at different microphone heights and these indicated negligible difference in the comparison between measured and predicted sound pressure levels with regard to a different height of the microphone. Hence, all testing for these investigations was carried out at one microphone height. As before, measurements were taken at 5 degree intervals over an arc of \pm 25 degrees. The maximum angle of the measurement arc was restricted by the angle of the fully open doors of the anechoic chamber. At the test frequency of $1 \mathrm{kHz}$ and for the size of apertures tested this measurement arc allowed both the dominant "line of sight" peak of each tested mode to be measured as well as much of the low amplitude "trough-like" behaviour that characteristically occurs 
spatially on either side of the dominant peak. Both the door surfaces and the ground outside the chamber were covered with thick foam to reduce errors from reflected sound waves. Fig. 6 shows a schematic diagram of the plan view of the experimental setup.

As described above, the loudspeaker was positioned within the plane $z=6.3$ $\mathrm{m}$, and two different source locations, below the centre line of the aperture (low) or above the centre line (high), were used to excite the axial modes $(1,0)$ and $(2,0)$ at cuton. Measurements were taken for the two modes with both the high and low source locations to establish if the results were affected by reflection from the ground plane, even through the surface was covered with absorbent material. Although there was an expected level difference in the results for the two different source locations for the same mode, there was no discernible difference in the comparisons between the measured and predicted sound pressure levels for the set of screens tested. Thus, all results discussed in the following section relate to the high source location. A signal generator was used to input a $1 \mathrm{kHz}$ sine wave to the loud speaker for all tests, which gave approximately 50 higher-order modes cut-on in the largest open aperture with no solid laths present. This was reduced to 19 cut-on modes for the smallest aperture size. All testing was carried out using one frequency of the source, for as demonstrated, in non-dimensional format the results for any particular higher order scattered mode of the open aperture are independent of frequency. Each test concentrated on the spatial scattered field of one particular higher-order mode. Thus, it was decided to test at a single frequency even with the presence of the periodic screen. Sound pressure level measurements were made in the $1 / 3$ octave band centred on $1 \mathrm{kHz}$. For all tests, the measured sound pressure level $1 \mathrm{~m}$ from the source was approximately $100 \mathrm{~dB}$. The measured background noise in the transmitted field was 
consistently $40 \mathrm{~dB}$ or less in normal conditions for each of the tests. For all values of aperture aspect ratio, the majority of the measured values of sound pressure level around the measurement arc were a minimum of $50 \mathrm{~dB}$ above the background noise level. However, the occasional reading near to the extremities of the measurement arc did drop below $50 \mathrm{~dB}$. As such, these values may have been affected by the background noise. To determine the sound transmission through the baffle plate itself, measurements of sound pressure level on the transmitted side were made with the aperture section of the baffle plate filled in with the same material and construction as the baffle plate thus making a solid plate. In this situation, the recorded level differences varied with microphone position on the arc, but were generally $10-15 \mathrm{~dB}$ below the levels recorded for the same microphone locations with the equivalent open aperture. The smallest difference of $10 \mathrm{~dB}$ occurred at the two extremities of the measurement arc. All measurements for all tests were repeated, with typically less than $1 \mathrm{~dB}$ difference in the results for all the measurement locations in the transmitted field and all of the screens tested. Measured sound pressure levels shown on the figures discussed in the next section are the average of two tests and in each case the source is located in the high position.

\section{Results}

Due to the practical restrictions of the internal dimensions of the anechoic chamber, only four different cut-on modes in the aperture were investigated experimentally. These were the first two axial modes, $(1,0)$ and $(2,0)$, where the nodal lines would be formed parallel to the laths. Also investigated were the $(0,1)$ axial 
mode, where the nodal lines would be perpendicular to the laths, and the $(2,1)$ tangential mode where the nodal lines are both parallel and perpendicular to the laths. The $(0,1),(1,0)$ and $(2,0)$ modes should ideally produce a spatially symmetric scattered field with the dominant section of the fields located on the centre of the aperture. However, for the $(2,1)$ mode the fields should be spatially non-symmetric with the dominant section off set from the aperture centre. Of significant interest was when the laths blocked the dominant direct 'line of sight' path from the source through the centre of the aperture to the transmitted field or when the laths cut across the nodal lines in the modal patterns. In each of these cases, the uncoupled mode assumption used in the estimate of the scattered field may no longer be valid. Also the presence of the multiple laths would increase the contribution from the evanescent waves that are unaccounted for in the uncoupled analysis. For each mode, measurements were made with various numbers of slits and laths and Table 1 summarises the configurations investigated. In each case, the slits and laths were equally spaced apart at $70 \mathrm{~mm}$ intervals resulting in a constant porosity of 0.5 . Also given in Table 1 is the number of cut-on modes in the equivalent open aperture. Previous analytical investigations [12] had established that 25 cut-on in-aperture modes was the minimum number required for the approximate approach to give acceptable results. As may be observed in Table 1, the aperture of aspect 4.35 did not satisfy the minimum criteria of 25 cut-on in-aperture modes, with only 19 modes cuton at $1 \mathrm{kHz}$. However, this aspect ratio was included in order to investigate the effect of insufficient cut-on modes on the results. As noted above, the presence of the laths would increase the contribution from the evanescent waves to the scattered field. As the number of modes cut-on in the aperture decreased, the contribution from the evanescent waves would be more significant. Hence, it might be expected that the 
differences between the estimate of the scattered field and the measured field would increase with a reduction in the number of in-aperture cut-on modes.

The transmission coefficients for the screens were calculated using equations (4) and (5). As the sound field was three dimensional in nature, transmission coefficients were calculated for both impingement planes and the resultant taken. As the source changes position for each mode, the transmission loss was recalculated as the impingement angles changed with the mode selected. This resulted in a transmission loss for modes $(1,0),(2,0)$ and $(0,1)$ of $3.01 \mathrm{~dB}$ and for mode $(2,1)$ of $2.37 \mathrm{~dB}$. As noted before the porosity of the screens was kept constant at a high value of 0.5 , resulting in small values of transmission loss at the test frequency of $1 \mathrm{kHz}$. Modelling the periodic screen as a mass law means the transmission loss would increase with frequency at the rate of $6 \mathrm{~dB}$ per octave, up to the point where diffraction effects occur due to the separation between the laths

Figs. 7 and 8 show the comparison, for the $(1,0)$ and $(2,1)$ respectively, between the measured data for the largest open aperture and the same aperture containing the screen with eight slits. In each figure the measured data for the wholly open aperture has had the relevant calculated transmission loss for the periodic screen subtracted from it to allow simple comparison with the periodic screen measurement. The $(1,0)$ mode is shown in Fig. 7 and this shows that the corrected open aperture measurement and the periodic screen measurement are within $1 \mathrm{~dB}$ of each other in the region around the origin. This increases to approximately $2 \mathrm{~dB}$ at the extremes of the measurement arc. As noted in the previous section, the accuracy of the measurements at the extremes of the arc may be questioned due to the presence of the anechoic chamber doors. For these measurements, seven laths were placed in the aperture, which meant that a lath was positioned in the centre of the aperture, directly 
in line with the 0 degree measurement point. For the $(1,0)$ mode of the aperture, without a screen present, the maximum sound pressure level will be at 0 degree, which represents the direct path from the source through the centre of the aperture. Thus, a lath was blocking the most dominant path from the source to the measuring points. However, as may be observed from Fig.7, the scattered field from the aperture with the screen maintained the same spatial characteristics as the open aperture. Similar results were obtained for the other two axial modes, $(2,0)$ and $(0,1)$ with again the greatest differences between the corrected open aperture measurement and the periodic screen measurements being at the extremes of the measurement arc. The spatial characteristics of both the open aperture and the aperture with the screen were the same, implying that as expected a simple reduction in the sound pressure level had occurred by introducing the screen regardless of the characteristics of the higher order mode. It should be noted that for both of these modes the direct path from the source, through the centre of the aperture to the measuring points, was not blocked by a lath.

Results for the tangential $(2,1)$ mode are shown in Fig. 8 and again the relevant transmission loss for the screen has been subtracted from the wholly open aperture measurement. For the $(2,1)$ mode the maximum response should occur at approximately minus 10 degrees, which is equivalent at $1 \mathrm{kHz}$ to $k \sin \phi=-3.2$, a location that represents the direct path from the source. The difference between the two sets of data is nearly always less than $1 \mathrm{~dB}$, with the peaks and troughs of the periodic screen measurements matching those in the open aperture measurement. Although the modal pattern is more complicated than for the axial modes, the spatial characteristics of both the open aperture and the aperture with a screen are the same, with no shift in the relative spatial position of the maxima or minima. Thus, it is reasonable to assume that the difference between the transmitted higher mode field 
sound pressure level for an open aperture and an aperture with a periodic screen inserted is simply the transmission loss determined from the equivalent mass layer based on plane wave analysis. Although it is well established that the mass layer transmission loss may be used for plane wave propagation through a screen in an aperture, it may not always be applicable to the higher-mode situation, when modes are present across the aperture.

To increase the aspect ratio while maintaining the same porosity for the aperture, more solid laths were added to the edges of the aperture, thus, blocking-in the outermost slits. This had the result of increasing the aspect ratio but reducing the number of laths and slits, as indicated in Table 1. Also this approach had the effect of changing the spatial pattern of the transmitted field relative to the fixed microphone measurement positions due to the equivalent open aperture parameter $2 b$ being reduced. Thus, it was not possible to easily compare the spatial characteristics of the same mode for two different sized apertures, as the positions were, in absolute terms, at different distances from the edge of the aperture. The effect of reducing the dimension $2 b$ may be seen from Fig. 9, in which the measured slit screen results for the $(2,0)$ mode for the six different aspect ratios tested are plotted against $k \sin \phi$ and aperture parameter $2 b$. Fig. 9 shows how a reduction in the aspect ratio of the aperture changes the absolute spatial pattern of the transmitted field but, relatively, the spatial pattern stays the same. The $(2,0)$ mode was selected to illustrate this point as it is an even-even mode which Ref. [9] considers to be more strongly coupled to the plane wave than even-odd modes and, hence, should exhibit the greatest error.

Figs. 10 to 12 show the comparison between predicted and measured sound pressure level for mode $(2,1)$ for slit screens with three different aspect ratios. This mode had the most complex modal pattern of the four modes investigated and, hence, 
the most complex spatial pattern. In each case the prediction for the wholly open aperture was determined using Eqs. (1), (2) and (3) and then the transmission loss for the screen, calculated using Eqs. (4) and (5) was subtracted from the estimated transmitted sound pressure level. Fig. 10 shows results for an aspect ratio of 1.52 with the aperture consisting of six laths and seven slits. Unlike the measured result for the 1.45 aspect ratio shown in Fig. 8, the central portion of the aperture is now a slit and not a solid lath. As with previous results the difference between measured and predicted data is greatest at the extremes of the measurement arc. The greatest difference is at $k \sin \phi=-4.7$, which corresponds to a measurement angle of minus 15 degrees. This was the value of $k \sin \phi$ where the greatest difference between measurement and prediction occurred for all the $(2,1)$ mode results. The difference at this value of $k \sin \phi$ was not present in either the $(2,0)$ mode or the $(0,1)$ mode results and the only observation that may be made is that the $(2,1)$ mode was the only tangential mode investigated. Results for the $(2,0)$ mode showed no particular difference in characteristics to those from the $(2,1)$ mode and, hence, no obvious difference between an even-even mode and the even-odd mode.

Fig. 11 shows the comparison for the aspect ratio 2.42 with the aperture consisting of four laths and five slits. Again the measured and predicted results follow the same trends. For the aspect ratio of 4.35, shown in Fig. 12, the number of modes cut-on in the aperture had reduced significantly to 19 , which is below the 25 mode limit suggested in [12] and, thus, the validity of the predicted sound pressure level is at the limit of the approach. This may be observed by comparing Fig. 12 to the predicted results in Figs. 10 and 11 that show smooth spatial responses with clearly identified maxima and minima, which are a feature of the scattered field from a higher mode when many modes are cut-on in the aperture. However, comparison between 
these and the predicted and measurement results shown in Fig. 12 shows a much reduced range on the data but the same spatial trends are evident even through the number of cut-on modes is less than the suggested lower limit for the approximation of 25. As with the other measurements, the greatest difference is at the extremes of angle.

The comparison between the four driven modes and the six aspect ratios is given in Table 2. In this table the average difference between measured and predicted sound pressure level is compared for each aspect ratio. When calculating the average difference, only data for the angles within \pm 20 degrees were used, as the accuracy of the measurement at the extremes of the arc is uncertain. From Table 2, it may be observed that the prediction is, in general, slightly greater than the measured transmitted level but in most cases less than $1 \mathrm{~dB}$. The two primary axial modes $(0,1)$ and $(1,0)$ have the smaller differences between measured and estimated sound pressure levels. These were the two modes with the simplest modal pattern. Also there is no discernable difference between the results for the $(0,1)$ and $(1,0)$ modes implying that the relative orientation of the laths and nodal lines had no noticeable effect. Similarly having a lath block the direct path from source to the microphone appears to have a minimal effect. As only four modes were considered, it is difficult to draw any overall conclusions from Table 2, apart from the observation that the differences increase with mode number and, hence, complexity of the modal pattern.

\section{Concluding comments}

Previously a decoupled scheme has been established for higher-order modes 
and it was shown analytically in Ref. [12] that for higher-order mode transmission the key parameters are the Helmholtz number of the incident wave, the aspect ratio and, to a lesser extent, the depth ratio of the aperture. Additionally, it was shown in Ref. [13] that if a point source is located far from the aperture and that if the acoustic pressure is measured at a small distance from the source then the acoustic pressure at any position in the transmitted field can be estimated using Eqs. (1), (2) and (3). This approach has been extended to an aperture with a periodic slit screen using the well established simple mass layer corrections. The estimated results remain valid within the frequency limits established in Refs. $[12,13]$. Thus, the aperture should be acoustically large such that at least 25 in-aperture modes are cut-on. If this is not the case then the incoming Helmholtz number should be increased. In effect, this sets the lower frequency limit for the analysis.

To validate this approach measurements have been reported of the scattered and transmitted pressure fields for higher-order modes propagating through a periodic slit screen located in a rectangular aperture in a rigid baffle. Comparison of the measured data for four different higher-order modes with and without the screen present shows that the difference in results is approximately equal to the equivalent mass layer transmission loss, based on plane wave impingement. Thus, although in the higher-order case, modes are set up across the aperture thickness, the mass layer representation for the periodic screen is still applicable. It should be noted that tests were carried out with a screen with a high level of porosity as the interest was in moving the laths on and off the direct line of sight from the source through the centre of the aperture to the receiver and hence determine the effect on the spatial response or directivity of the higher mode. Estimates of the transmitted field and the measured field for different modes were compared and good comparison was found, with the 
exception of the extremes of the measurement arc. The reliability of the measured data at the extremes of the measurement arc was uncertain due to the constraints on the baffle plate installation. Thus, it may be concluded that the uncoupled higherorder mode analysis can be extended to apertures containing simple periodic slit screens while still obtaining acceptable directivity estimates. Although the contribution from evanescent waves would have increased with the presence of the laths in the screens, good agreement was found between predicted and measured sound pressure levels provided the lower frequency limit of 25 in-aperture cut-on modes was maintained.

In summary, estimates of the scattered and transmitted fields through a periodic screen have been calculated using uncoupled higher mode analysis to determine the sound pressure level transmitted through an open aperture of the equivalent area as the periodic screen. The estimate of the sound pressure level is then simply corrected by the calculated transmission loss of the screen. This is a more straightforward approach to higher-order mode transmission through a periodic screen than the Fresnel-Kirchhoff diffraction technique for slit screen devices used by others. It also overcomes some of the frequency limitations of that technique. For the uncoupled mode approach to be used to estimate the performance of a periodic slit screen over a wide frequency range, the response for each higher-order mode in the range of interest could be calculated and the modal responses summed to obtain the overall frequency behaviour. In practical engineering terms, for a given frequency range of interest, the spatial distribution of the scattered and transmitted pressure field through a slit-lath aperture could be predicted by calculating the contribution of each mode and then summing all the modes, over the frequency range of interest, to obtain the whole field prediction. If required, this spatial distribution of pressure can be 
summed to produce a prediction of the total radiated sound power through the aperture.

\section{References}

1. A. Sauter, Jr., W.W. Soroka. Sound transmission through rectangular slots of finite depth between reverberant rooms. J Acoust Soc Am 1970; 47:5-11.

2. M.C. Gromperts. The "sound insulation" of circular and slit shaped apertures. Acustica 1964; 14:1-16.

3. M.C. Gromperts, T. Kihlman. The sound transmission loss of circular and slitshaped apertures in walls. Acustica 1967; 18:144-150.

4. F.P. Mechel. The acoustic sealing of holes and slits in walls. J Sound Vibr 1986; 111:297-336.

5. P.M. Morse, K.U. Ingard. Theoretical Acoustics. Princeton University Press, Princeton, NJ, 1986. pp. 492-521 (Chapter 9).

6. K. Honga, H. Serizawa. Diffraction of an acoustic plane wave by a rectangular hole in a infinitely large rigid screen. J Acoust Soc Am 1999; 106:29-35.

7. A.D. Pierce, R.O. Cleveland, M. Zampoli. Radiation impedance matrices for rectangular interfaces within rigid baffles: calculation methodology and applications. J Acoust Soc Am 2002; 111:672-684.

8. H.H. Park, H.J. Eom. Acoustic scattering from a rectangular aperture in a thick hard screen. J Acoust Soc Am (Letters) 1997; 101:595-598.

9. F. Sgard, H. Nelisse, N. Atalla. On the modelling of the diffuse fields sound transmission loss of finite apertures. J Acoust Soc Am 2007; 122:302-313. 
10. N. Trompette, J.L. Barbry, F. Sgard, H. Nelisse. Sound Transmission loss of rectangular and slit-shaped apertures: Experimental results and correlation with modal model. J Acoust Soc Am 2009; 125:31-41.

11. D.J. Oldham, X Zhao. Measurement of the sound transmission loss of circular and slit-shaped apertures in rigid walls of finite thickness by intensimetry. J Sound Vibr 1993; 161:119-135.

12. J. L. Horner, K. S. Peat. Approximations for the scattered field potential from higher mode transmission in rectangular apertures. J Acoust Soc Am 2006; 119:3568-3576.

13. J.L. Horner, K.S. Peat. Higher mode sound transmission from a point source through a rectangular aperture. J Acoust Soc Am 2011; 129:5-11.

14. L. Cremer, H. Muller. Principles and applications of room acoustics (T.J. Schultz, Trans.), Vol. 2. Applied Science Publishers, London; 1982.

15. C. Wassilieff. Improving the noise reduction of picket barriers. J Acoust Soc Am $1988 ; 84: 645-650$.

16. E.B. Viveiros, B.M. Gibbs, S.N.Y. Gerges. Measurement of sound insulation of acoustic louvres by an impulse method. Appl Acoust 2002; 63:1301-1313.

17. J.L. Horner, R. Lyons. Approximate method for determining the optimal position for noise sources near rectangular apertures. Proc Inst of Acoust 2003; 25:204211. 


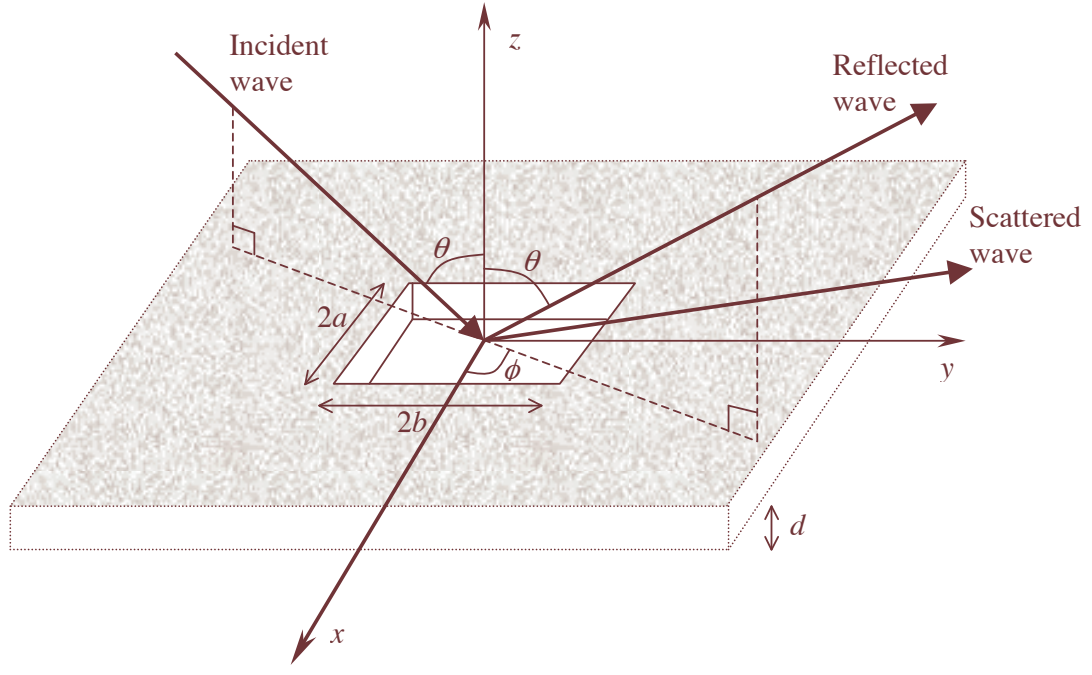

Fig. 1. Sketch of the aperture. 


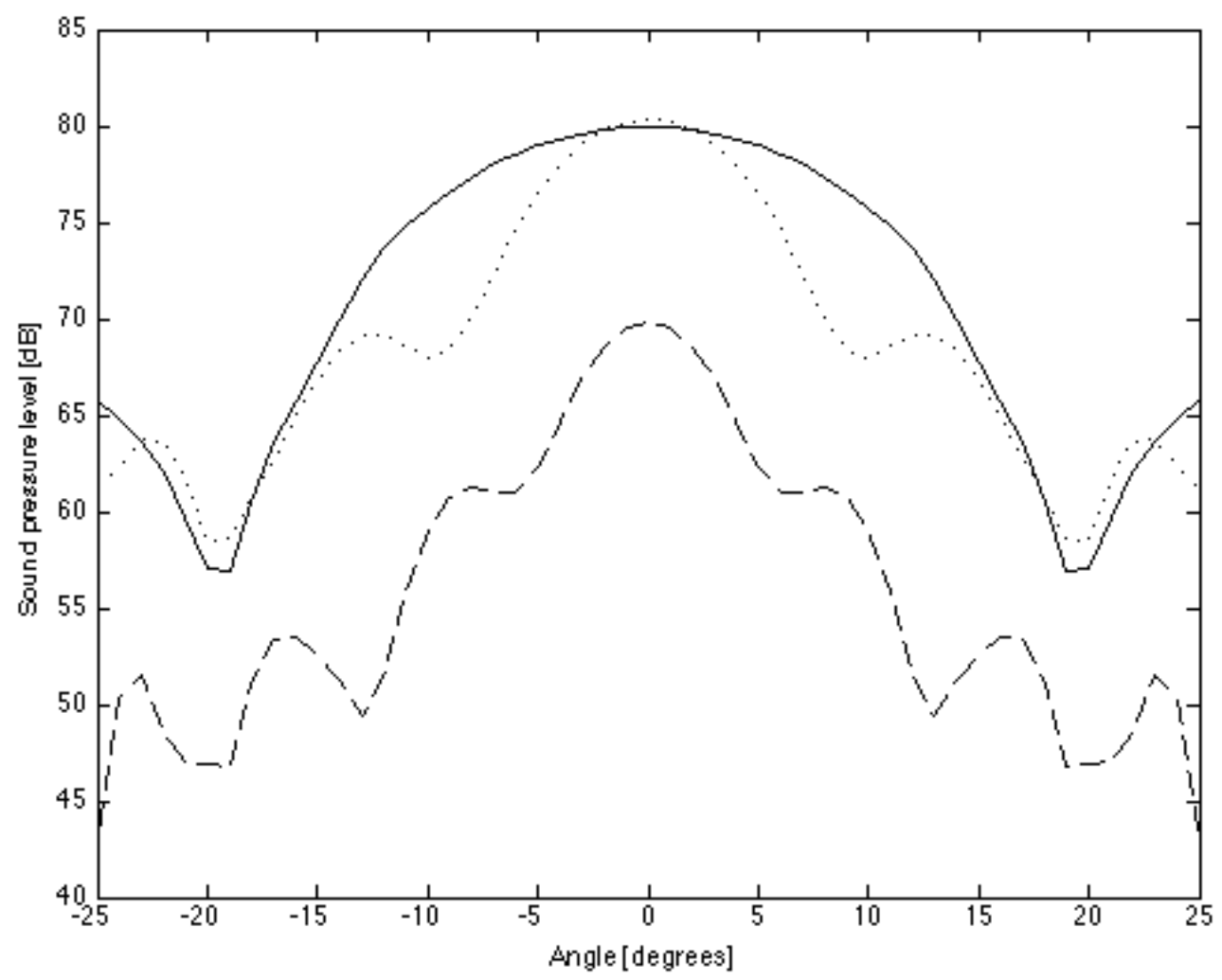

Fig. 2. Scattered Sound pressure level $[\mathrm{dB}]$ for the $(2,0)$ mode at $4.73 \mathrm{~m}$; _ $1 \mathrm{kHz}, \ldots 2$

$$
\mathrm{kHz},--3 \mathrm{kHz}) \text {. }
$$




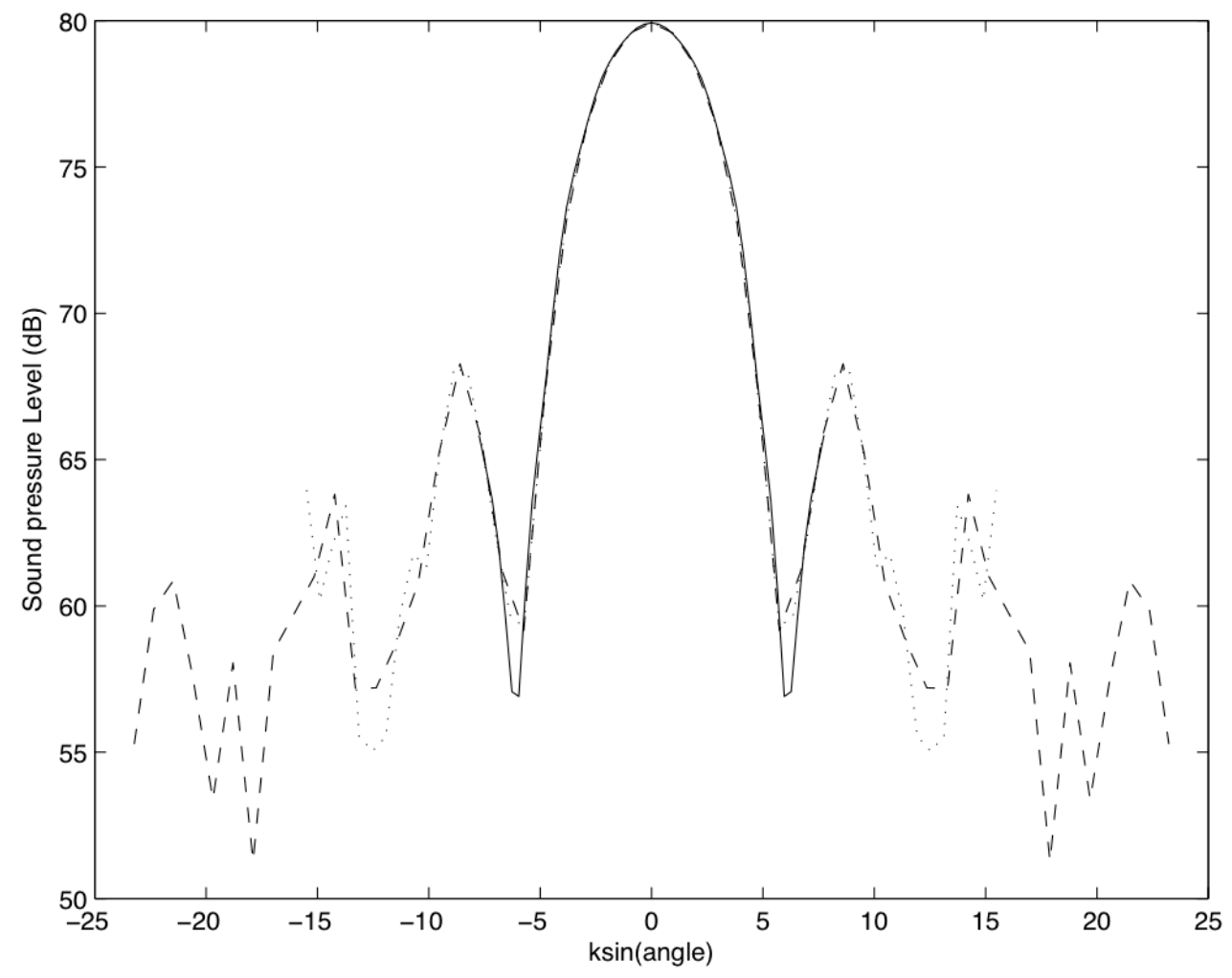

Fig. 3. Scattered field Sound pressure level $[\mathrm{dB}]$ for the $(2,0)$ mode; _ $1 \mathrm{kHz}, \ldots 2 \mathrm{kHz}$, - - $3 \mathrm{kHz})$. 


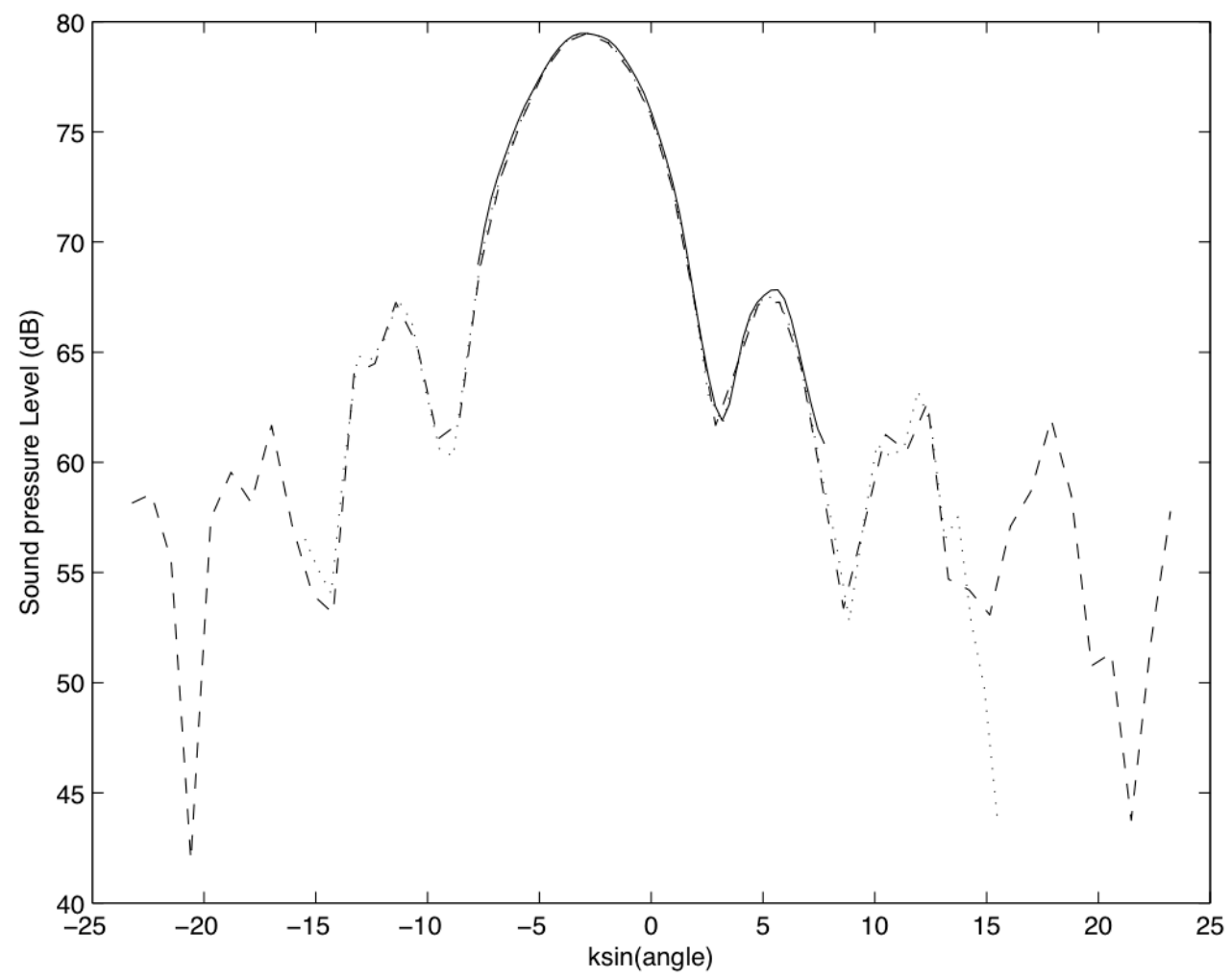

Fig. 4. Scattered field Sound pressure level $[\mathrm{dB}]$ for the $(2,1)$ mode; _ $1 \mathrm{kHz}, \ldots 2 \mathrm{kHz}$, - - $3 \mathrm{kHz})$. 


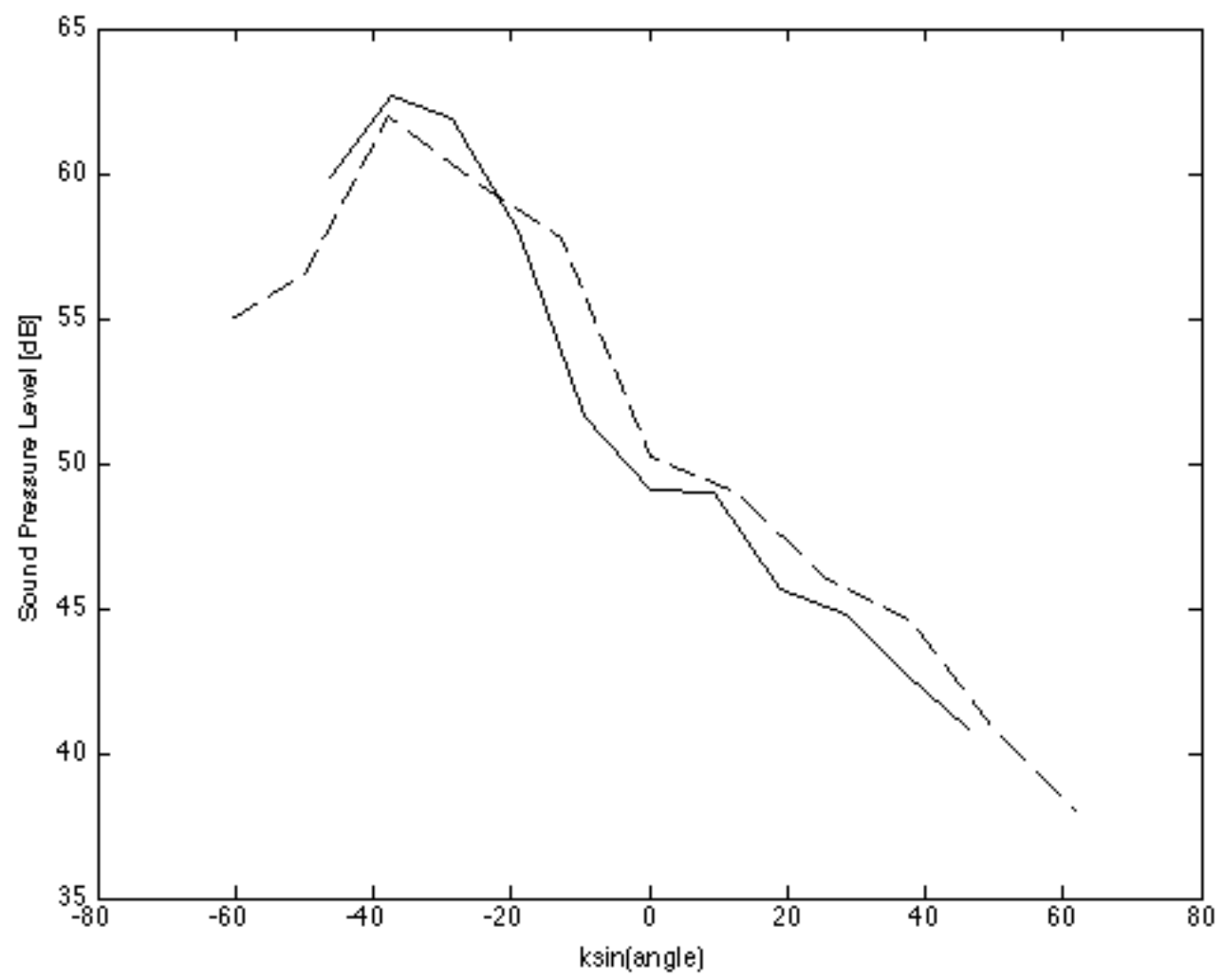

Fig. 5. Measured Sound pressure level $[\mathrm{dB}]$ for the $(2,1)$ mode; __ $6 \mathrm{kHz},---8 \mathrm{kHz})$. 


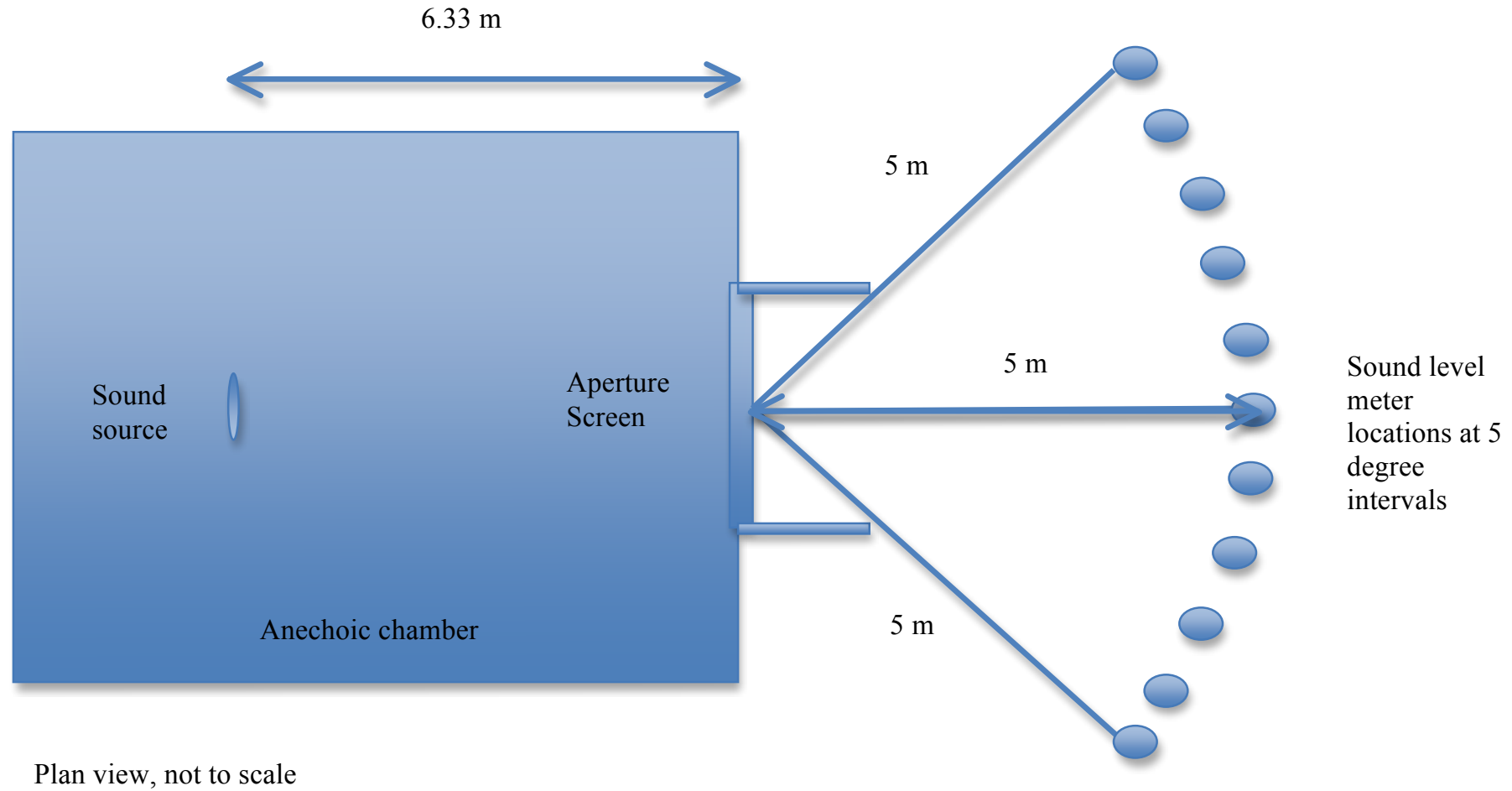

Fig. 6. Schematic representation of the experimental setup. 


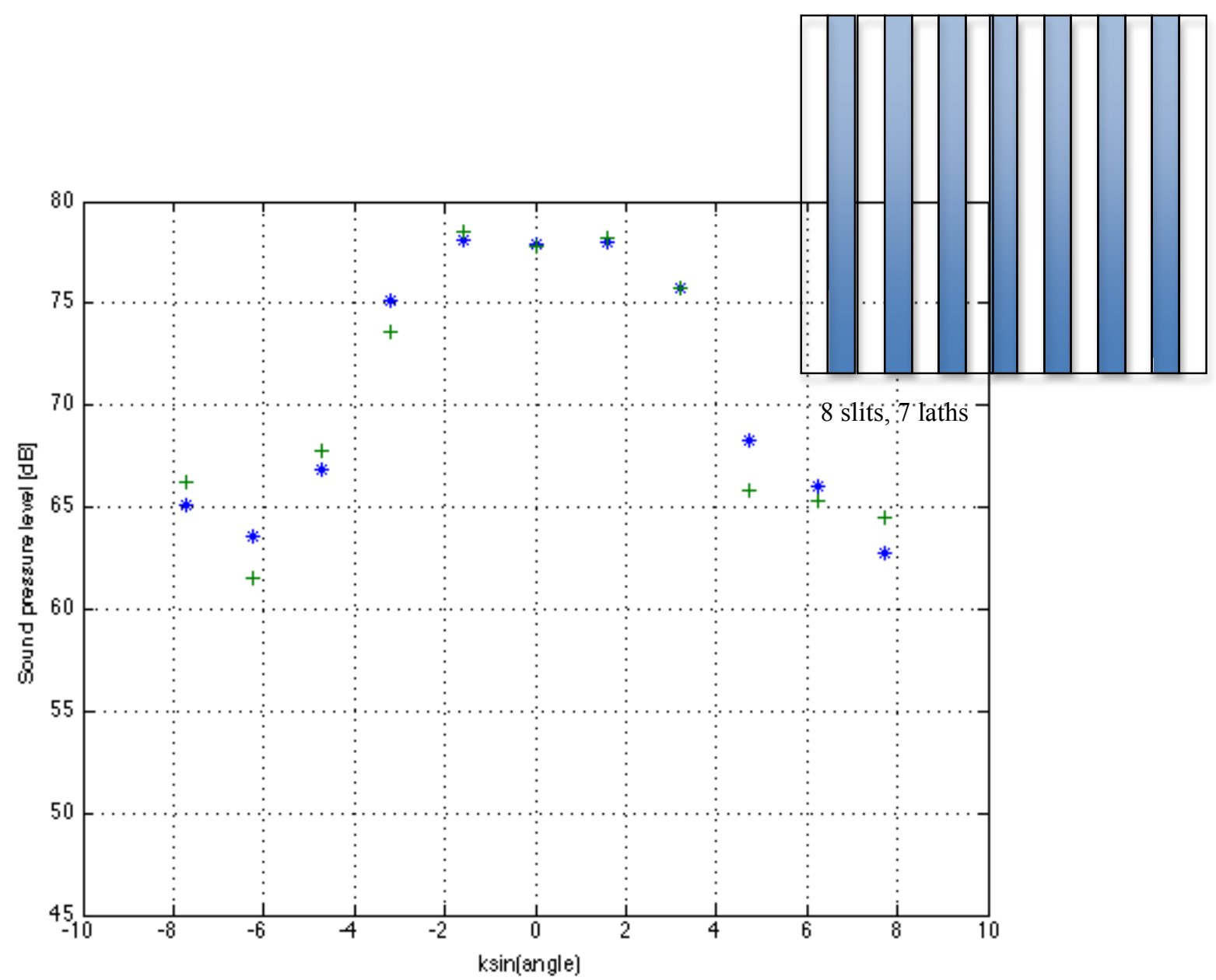

Fig. 7. Measured Sound pressure level $[\mathrm{dB}]$ in the transmitted field for the $(1,0)$ mode and an Aspect Ratio of 1.45: + Corrected Open Aperture; * Periodic Screen. 


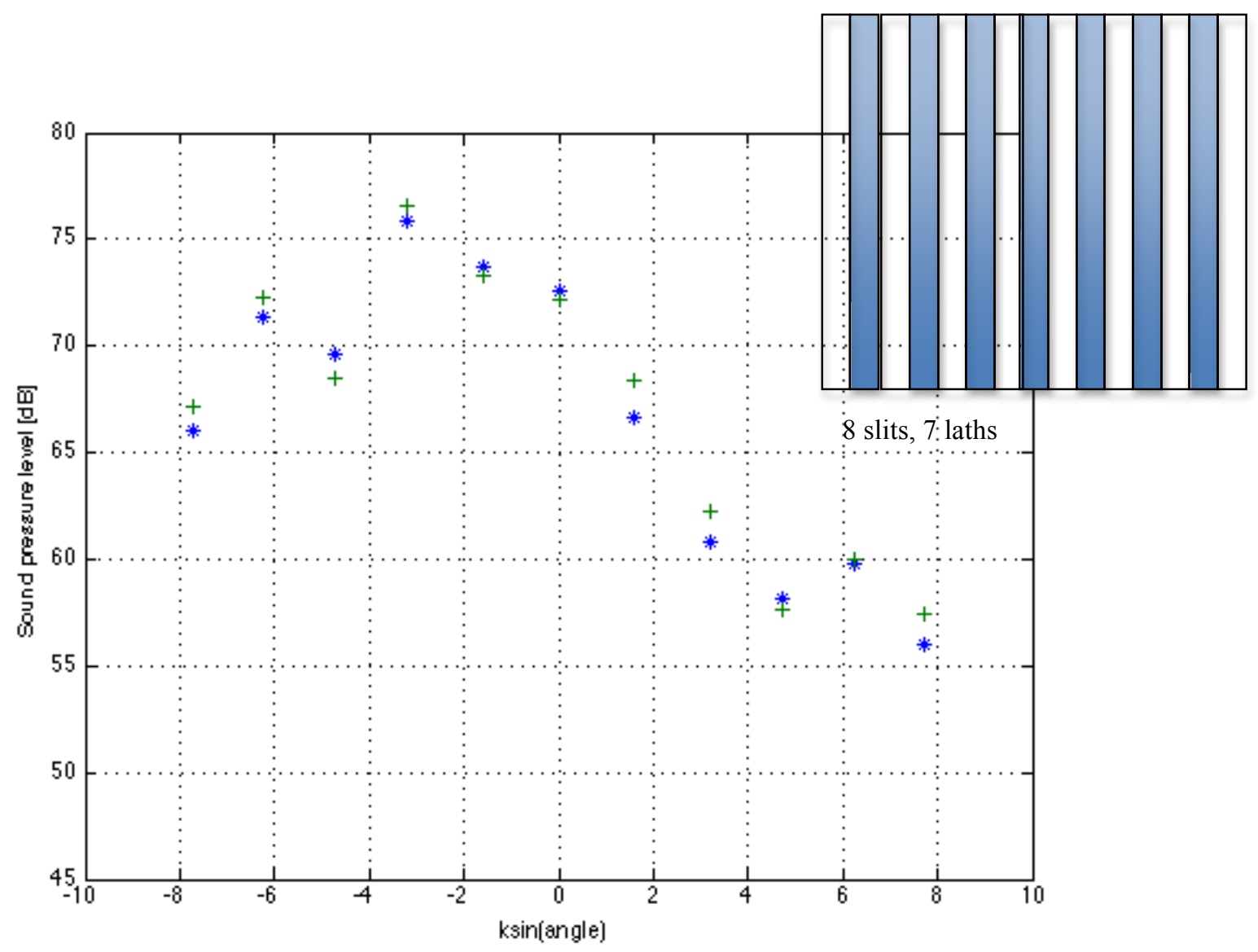

Fig. 8. Measured Sound pressure level $[\mathrm{dB}]$ in the transmitted field for the $(2,1)$ mode and an Aspect Ratio of 1.45: + Corrected Open Aperture; * Periodic Screen. 


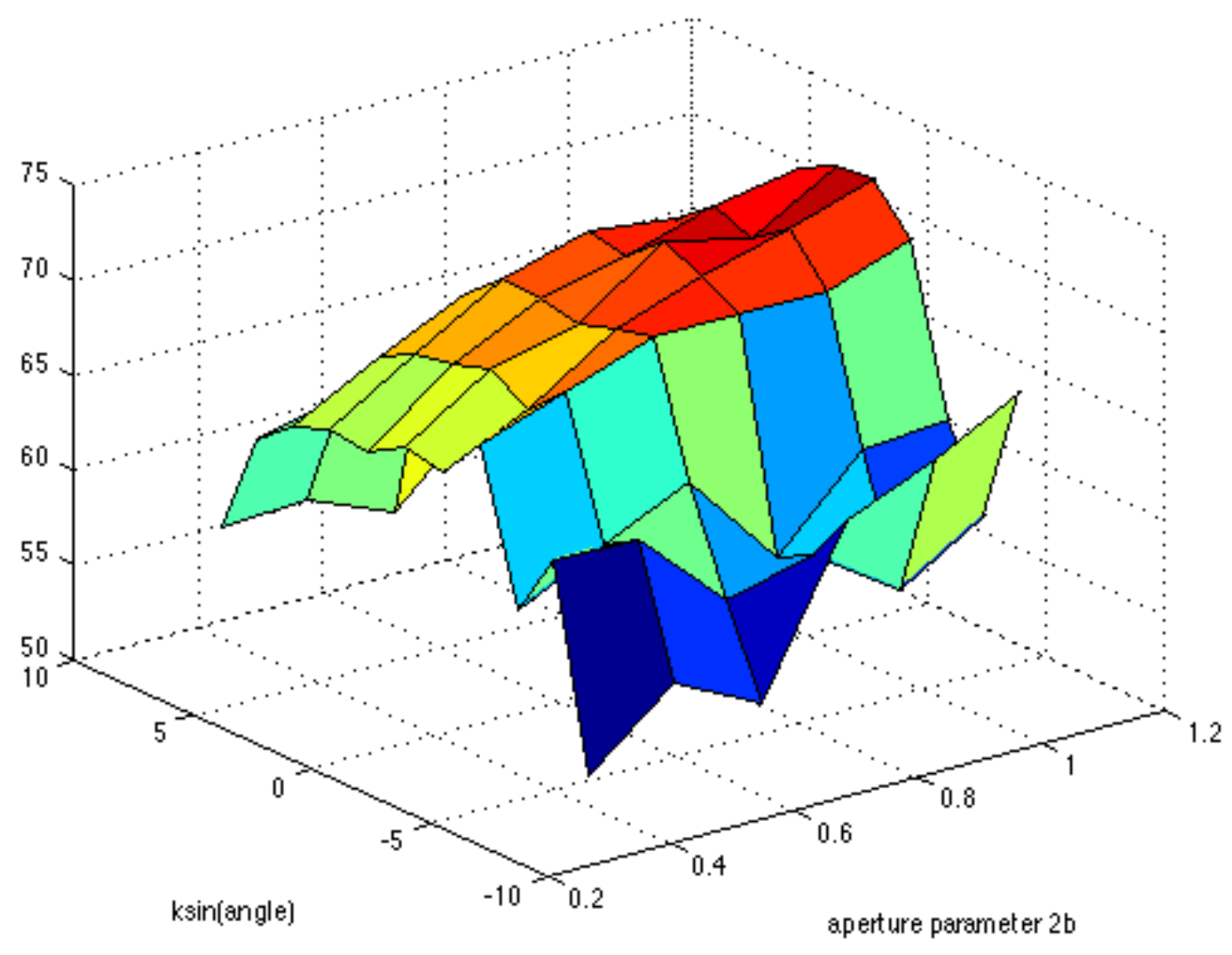

Fig. 9. Measured Sound pressure level $[\mathrm{dB}]$ in the transmitted field for the $(2,0)$ mode. 


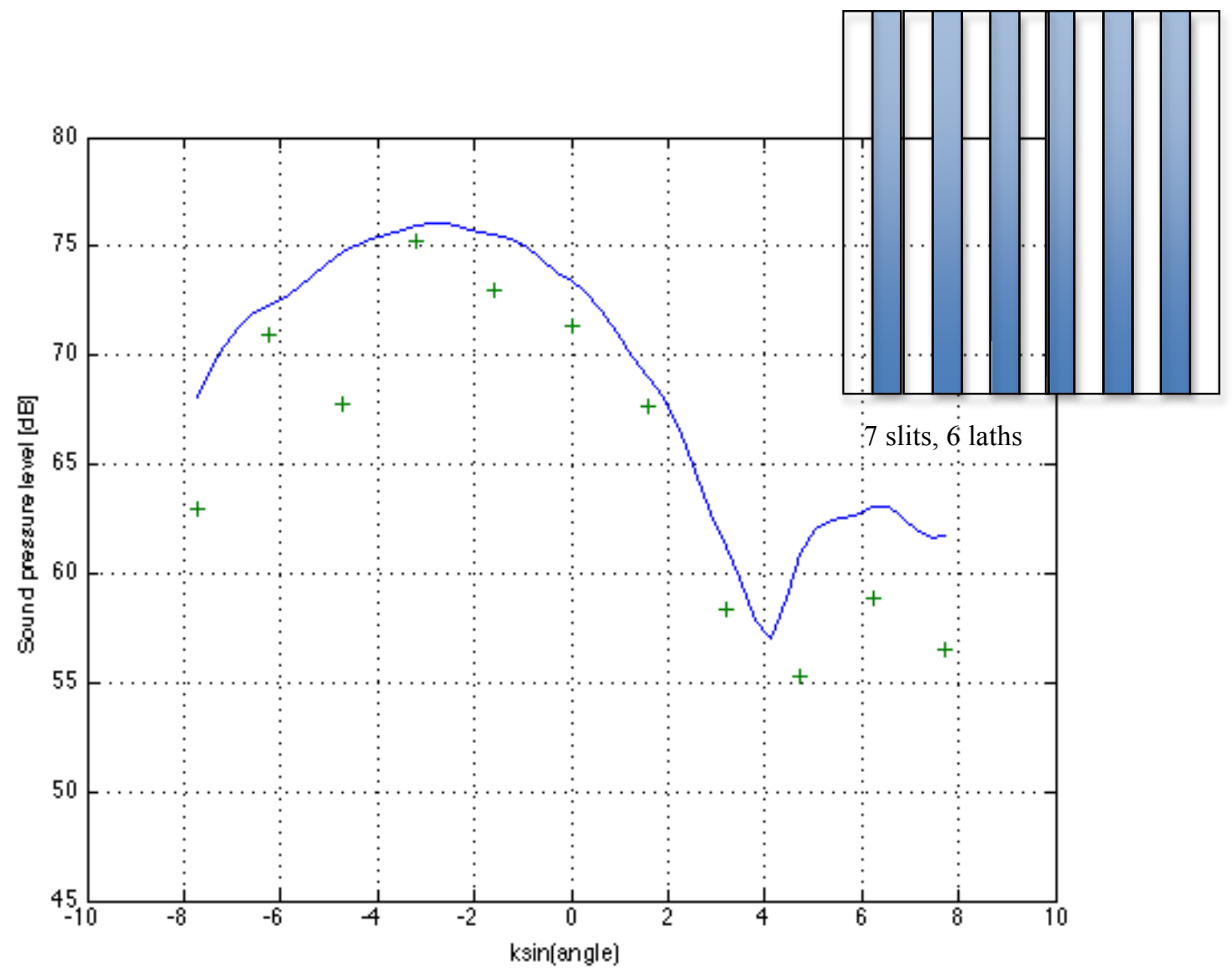

Fig. 10. Sound pressure level in the transmitted field for the $(2,1)$ mode and an Aspect Ratio of 1.52: + Experimental; --- Theoretical. 


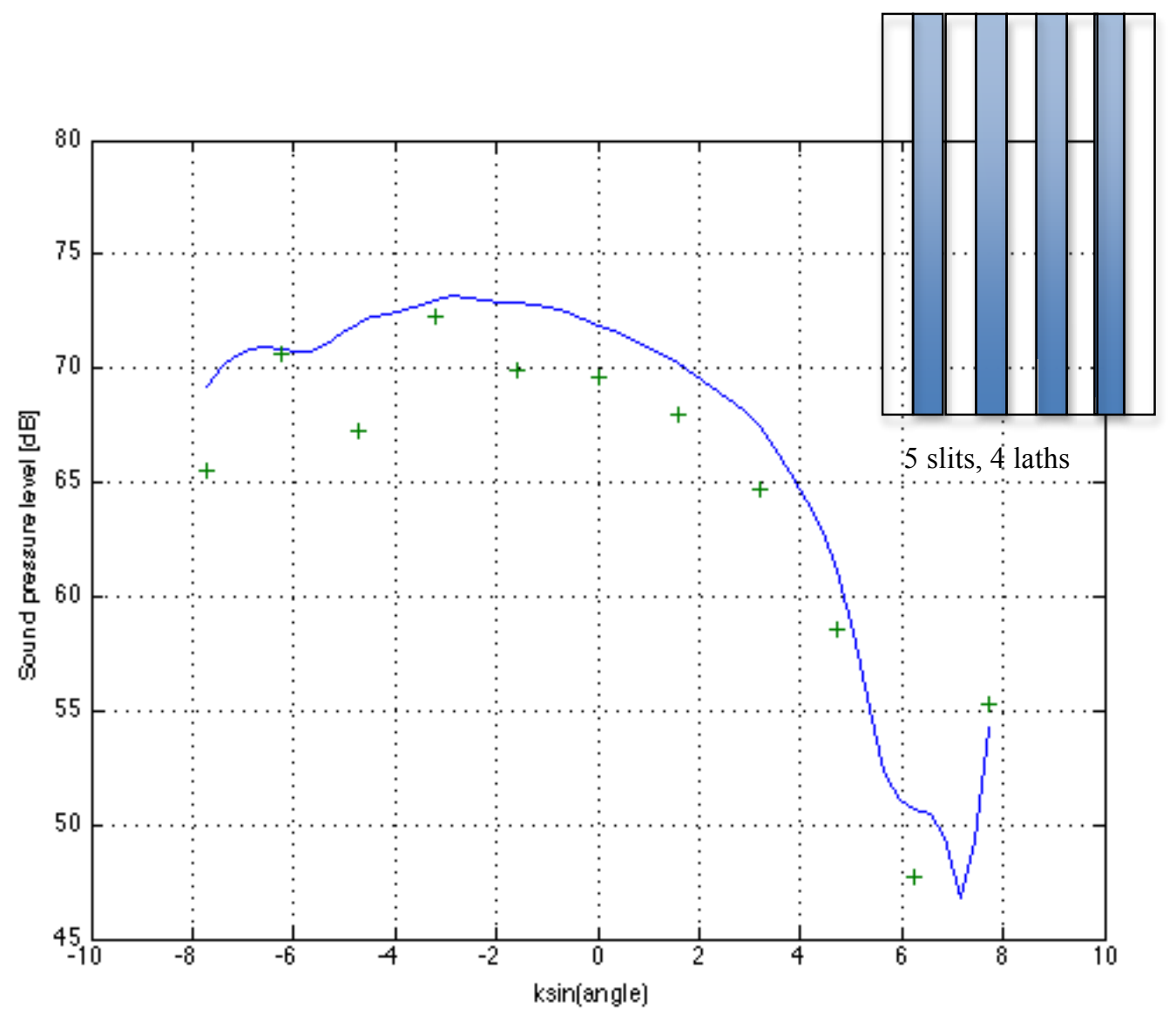

Fig. 11. Sound pressure level in the transmitted field for the $(2,1)$ mode and an Aspect Ratio of 2.42: + Experimental; --- Theoretical. 


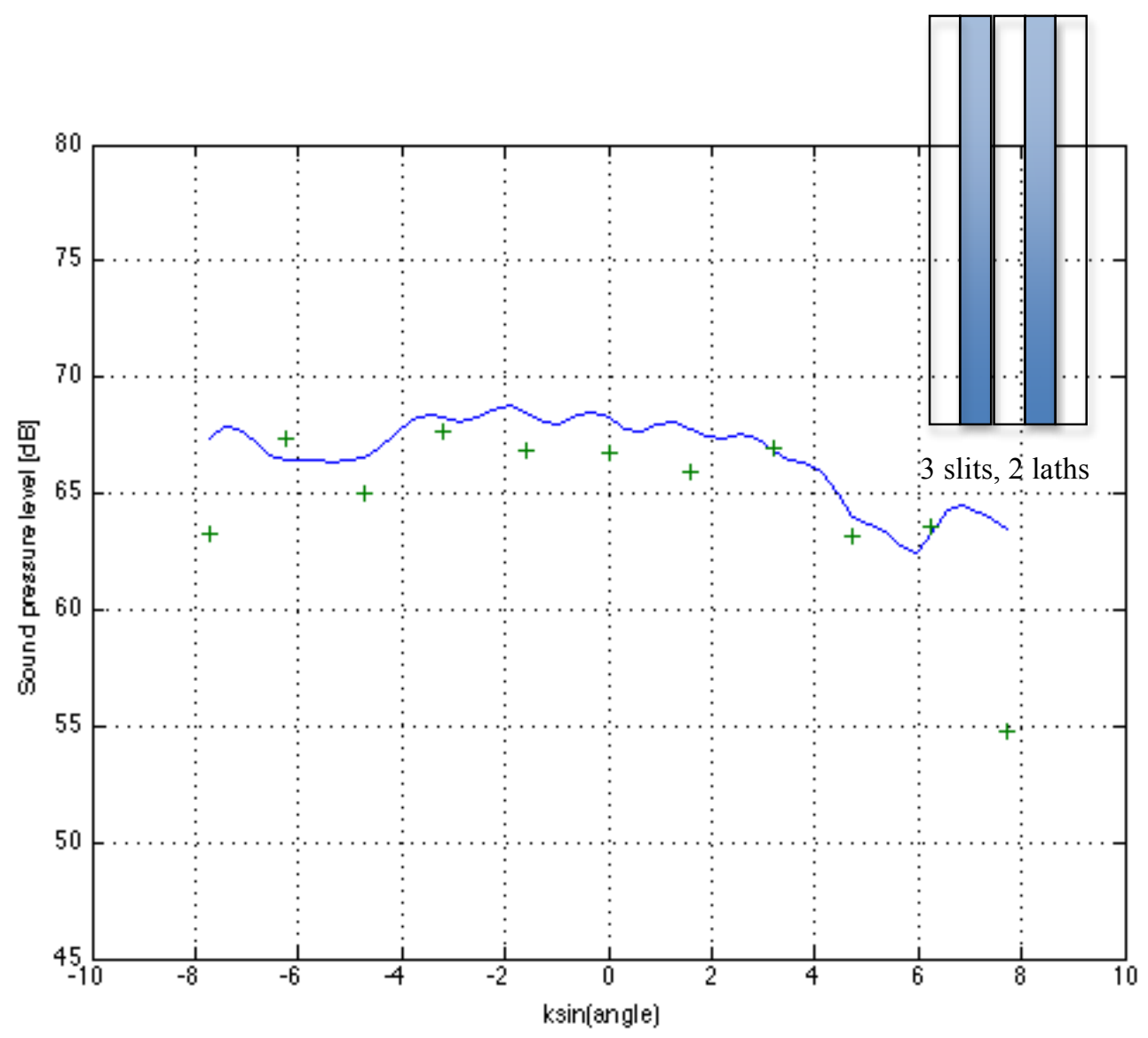

Fig. 12. Sound pressure level in the transmitted field for the $(2,1)$ mode and an Aspect Ratio 4.35: + Experimental; --- Theoretical. 
Table 1

Aperture configurations investigated experimentally.

\begin{tabular}{ccccc}
\hline $\begin{array}{l}\text { Aperture } \\
\text { dimension, 2b, } \\
(\mathrm{m})\end{array}$ & $\begin{array}{l}\text { Number of } \\
\text { slits }\end{array}$ & $\begin{array}{l}\text { Number of } \\
\text { laths }\end{array}$ & Aspect Ratio & $\begin{array}{l}\text { Number of } \\
\text { cut-on modes } \\
\text { at 1 kHz }\end{array}$ \\
\hline 0.35 & 3 & 2 & 4.35 & 19 \\
0.49 & 4 & 3 & 3.11 & 25 \\
0.63 & 5 & 4 & 2.42 & 32 \\
0.77 & 6 & 5 & 1.98 & 38 \\
0.91 & 7 & 6 & 1.52 & 44 \\
1.05 & 8 & 7 & 1.45 & 50 \\
\hline
\end{tabular}


Table 2

Average difference in $\mathrm{dB}$ between measured and estimated SPL.

\begin{tabular}{lcccc}
\hline $\begin{array}{l}\text { Aperture dimension, } \\
2 b,(\mathrm{~m})\end{array}$ & Mode $(0,1)$ & Mode $(1,0)$ & Mode $(2,0)$ & Mode $(2,1)$ \\
\hline 0.35 & -0.17 & -0.70 & -2.45 & -0.72 \\
0.49 & -1.18 & -0.95 & -2.67 & -1.30 \\
0.63 & -0.44 & -1.63 & -1.32 & -1.83 \\
0.77 & -0.63 & -0.95 & -2.97 & -1.92 \\
0.91 & -0.54 & 0.13 & -1.17 & -3.08 \\
1.05 & -1.43 & 0.13 & -0.68 & -2.19 \\
\hline
\end{tabular}

